\title{
Generalized Planar Turán Numbers
}

\author{
Ervin Győri* \\ Alfréd Rényi Institute of Mathematics \\ Budapest, Hungary \\ Central European University \\ Budapest, Hungray \\ gyori.ervin@renyi.mta.hu \\ Nika Salia*† \\ Alfréd Rényi Institute of Mathematics \\ Budapest, Hungary \\ Central European University \\ Budapest, Hungray \\ salia.nika@renyi.hu
}

\author{
Addisu Paulos \\ Addis Ababa University \\ Addis Ababa Ethiopia \\ Central European University \\ Budapest, Hungary \\ addisu_2004@yahoo.com \\ Casey Tompkins ${ }^{\ddagger}$ \\ Institute for Basic Science (IBS) \\ Daejeon, Republic of Korea \\ Karlsruhe Institue of Technology \\ Karlsruhe, Germany \\ ctompkins496@gmail.com
}

\author{
Oscar Zamora* \\ Central European University \\ Budapest, Hungary \\ Universidad de Costa Rica \\ San José, Costa Rica \\ oscar.zamoraluna@ucr.ac.cr
}

Submitted: May 22, 2020; Accepted: Sep 23, 2021; Published: Nov 19, 2021

(C) The authors. Released under the CC BY license (International 4.0).

\begin{abstract}
In a generalized Turán problem, we are given graphs $H$ and $F$ and seek to maximize the number of copies of $H$ in an $n$-vertex graph not containing $F$ as a subgraph. We consider generalized Turán problems where the host graph is planar. In particular, we obtain the order of magnitude of the maximum number of copies of a fixed tree in a planar graph containing no even cycle of length at most $2 \ell$, for all $\ell, \ell \geqslant 1$. We also determine the order of magnitude of the maximum number of cycles of a given length in a planar $C_{4}$-free graph. An exact result is given for the maximum number of 5 -cycles in a $C_{4}$-free planar graph. Multiple conjectures are also introduced.
\end{abstract}

Mathematics Subject Classifications: 05C10, 05C35

*Supported by the National Research, Development and Innovation Office, NKFIH, grant K132696.

†Supported by Shota Rustaveli National Science Foundation of Georgia SRNSFG, grant DI-18-118.

${ }^{\ddagger}$ Supported by IBS-R029-C1. 


\section{Introduction}

For a given family of graphs $\mathcal{F}$, we say that a graph is $\mathcal{F}$-free if it contains no $F \in \mathcal{F}$ as a subgraph. The classical extremal function $\operatorname{ex}(n, \mathcal{F})$ is defined to be the maximum number of edges possible in an $n$-vertex $\mathcal{F}$-free graph. This function naturally generalizes to a setting where, rather than edges, we maximize the number of copies of a given graph $H$ in an $n$-vertex $\mathcal{F}$-free graph. Following Alon and Shikhelman [2] (see also [3]), we denote this more general function by $\operatorname{ex}(n, H, \mathcal{F})$. In the case that $\mathcal{F}=\{F\}$, we denote the generalized extremal function $\operatorname{ex}(n, H, \mathcal{F})$ by $\operatorname{ex}(n, H, F)$ and we refer to $\mathcal{F}$-free graphs simply as $F$-free.

Problems of this type have a long history beginning with a result of Zykov [38] (and later independently Erdös [11]) who determined the value of $\operatorname{ex}\left(n, K_{r}, K_{t}\right)$ for any pair of cliques. After these initial results a variety of other results of this type were obtained, perhaps the most well-known of which being the determination of $\operatorname{ex}\left(n, C_{5}, C_{3}\right)$ by Hatami, Hladký, Král, Norine and Razborov [22] and independently by Grzesik [17]. Many other results about generalized extremal numbers have also been obtained (see, for example $[4$, $14,32,33])$.

Another natural generalization of the Turán extremal function is to the setting where the host graph is taken to be something other than $K_{n}$. Examples include the Zarankiewicz problem [37] wherein the host graph is taken to be a complete bipartite graph $K_{\frac{n}{2}}, \frac{n}{2}$, or extremal problems on the hypercube $Q_{n}$ initiated by Erdős [12].

More recently, extremal problems have been considered where the host graph is taken to be a planar graph. For a given graph $F$, let us denote the maximum number of edges in an $n$-vertex $F$-free planar graph by $\operatorname{ex}_{\mathcal{P}}(n, F)$. This topic was initiated by Dowden in [9] who determined $\operatorname{ex}_{\mathcal{P}}\left(n, C_{4}\right)$ and $\operatorname{ex}_{\mathcal{P}}\left(n, C_{5}\right)$. A variety of other forbidden graphs $F$ including stars, wheels and fans were considered by Lan, Shi and Song [29]. The case of theta graphs was considered in Lan, Shi and Song [30], and the case of short paths was considered by Lan and Shi in [28]. Some closely related anti-Ramsey problems were considered in [31] and [6].

Another direction of research which has been considered is maximizing the number of copies of a given graph in an $n$-vertex planar graph. Hakimi and Schmeichel [21] determined the maximum number of triangle and $C_{4}$ copies possible in a planar graph. These results were extended by Alon and Caro [1] who determined the maximum number of $K_{1, t}$ and $K_{2, t}$ copies. Alon and Caro [1] and independently Wood [35] proved that the maximum number of $K_{4}$ copies in a planar graph is $n-3$. Resolving a conjecture attributed to Perles in [1], Wormald [36] proved that every 3-connected graph $H$ occurs at most $c_{H} n$ times in an $n$-vertex planar graph for some constant $c_{H}$ depending on $H$ (this result was proved again in a different way by Eppstein [10]). A simple argument shows that graphs with at least 3 vertices which are not 3-connected can occur at least quadratically many times in a planar graph. Thus the preceding result of Wormald and Eppstein provides a characterization of graphs which can occur at most $O(n)$ times in a planar graph. Recently, resolving a conjecture of Hakimi and Schmeichel [21], the maximum number of 5 -cycles in a planar graph was determined in [18]. Similarly, the maximum number of 
paths of length three was determined in [19]. More recently, asymptotic results have been obtained for some longer paths and cycles by Ghosh et al. [16] and Cox and Martin [7, 8]. Matolcsi and Nagy [34] have also obtained results in the outerplanar setting.

It is interesting to note that the problem of maximizing the number of $H$ copies in a planar graph is in some sense a special case of the problem of Alon and Shikelman [2]. Indeed, for a given graph $H$, and the collection $\mathcal{F}$ of subdivisions of $K_{5}$ and $K_{3,3}$, it follows from Kuratowski's [26] theorem that $\operatorname{ex}(n, H, \mathcal{F})$ is equal to the maximum number of $H$ copies in an $n$-vertex planar graph.

In this paper we will consider a common generalization of the types of problems mentioned above. To this end, let $\operatorname{ex}_{\mathcal{P}}(n, H, \mathcal{F})$ denote the maximum number of copies of $H$ possible in an $n$-vertex planar graph containing no graph $F \in \mathcal{F}$ as a subgraph (we write simply $\operatorname{ex}_{\mathcal{P}}(n, H, F)$ in the case $\left.\mathcal{F}=\{F\}\right)$. In particular, the problems considered in the preceding paragraphs correspond to the special cases of $\operatorname{ex}_{\mathcal{P}}\left(n, K_{2}, F\right)$ and $\operatorname{ex}_{\mathcal{P}}(n, H, \varnothing)$, for given graphs $F$ and $H$.

\section{Notation and Results}

For a graph $G$, we denote the vertex set and edge set of $G$ by $V(G)$ and $E(G)$, respectively. Also we let $v(G)=|V(G)|$ and $e(G)=|E(G)|$. For a vertex $v \in V(G)$, the degree of $v$ is denoted by $d(v)$, and the minimum degree of the graph $G$ is denoted by $\delta(G)$. We use the notation $C_{t}$ and $K_{t}$ respectively for the cycle and complete graph of order $t$. We denote the path on $t$ vertices ( $t$-vertex path) by $P_{t}$. For a graph $G$ and an independent set $S \subseteq V(G)$, the graph obtained by blowing $S$ up by $t$ is the graph formed by replacing each vertex $v \in S$ with $t$ vertices each with the same neighbors as $v$. Note that in all of the results and conjectures we discuss the constants hidden in the asymptotic notation are allowed to depend on the graphs appearing in the $\operatorname{ex}_{\mathcal{P}}$ function.

In the general case, Eppstein [10] asked whether for all $H$, the maximum number of copies of $H$ possible in a minor-closed family of $n$-vertex graphs is an integer power of $n$. We state a restricted version of his problem as a conjecture in the planar case, and later generalize it further.

Conjecture 1. For every graph $H$, there exists a non-negative integer $k$, such that

$$
\operatorname{ex}_{\mathcal{P}}(n, H, \varnothing)=\Theta\left(n^{k}\right) .
$$

Remark 2. Conjecture 1 has now been resolved in a more general setting of subgraphs in an arbitrary surface by Huynh, Joret and Wood [23] (see also [24] for further work in general settings).

We verify this conjecture in the case of trees. Moreover, we prove a generalization in the case the graph is assumed to have no short even cycles. To state our results we require some notation. 
Definition 3. For a graph $H$ and an integer $i, i \geqslant 1$, let $\beta_{i}(H)$ be the maximum number of components in an induced subgraph of $H$ containing only two types of components: (1) isolated vertices which have degree one in $H$ and (2) paths of length $i-1$ consisting only of vertices of degree two from $H$.

In particular in the case when $i=1$, we are interested in the maximum size of an independent set in the graph consisting of vertices of degree at most 2 . For simplicity, we let $\beta(G):=\beta_{1}(G)$ for any graph $G$. Then we have the following result for trees.

Theorem 4. Let $T$ be a tree, then

$$
\exp _{\mathcal{P}}(n, T, \varnothing)=\Theta\left(n^{\beta(T)}\right) .
$$

For any graph $H$, let $\alpha(H)$ be the independence number of $H$. In the general case we have the following upper bound.

Theorem 5. Let $H$ be any graph, then

$$
\operatorname{ex}_{\mathcal{P}}(n, H, \varnothing)=O\left(n^{\alpha(H)}\right) .
$$

This theorem will follow as an immediate consequence of results we prove about degenerate graphs in Section 3. As a corollary of Theorem 5 we obtain the order of magnitude of the maximum number of cycles (note that this result was already obtained by Hakimi and Schmeichel in [21]).

Corollary 6. For all $k \geqslant 3$, we have

$$
\operatorname{ex}_{\mathcal{P}}\left(n, C_{k}, \varnothing\right)=\Theta\left(n^{\lfloor k / 2\rfloor}\right) .
$$

The lower bound is attained by taking a cycle $C_{k}$ and blowing up a maximum-size independent set by $\lfloor 2 n / k\rfloor-1$. Note that the constant in the asymptotic notation may depend on $k$, and this construction contains asymptotically $\left(\frac{2 n}{k}\right)^{\lfloor k / 2\rfloor}$ copies of $C_{k}$.

Next we consider the case when the set of forbidden graphs is nonempty. In this case, we pose a conjecture which generalizes Conjecture 1 .

Conjecture 7. For all finite sets of graphs $\mathcal{F}$ and for all graphs $H$, we have

$$
\operatorname{ex}_{\mathcal{P}}(n, H, \mathcal{F})=\Theta\left(n^{k}\right),
$$

for some integer $k$.

We consider a variation of Theorem 4 for the case when $C_{4}, C_{6}, \ldots, C_{2 \ell}$ are forbidden. We prove the following.

Theorem 8. For any tree $T$, we have

$$
\exp _{\mathcal{P}}\left(n, T,\left\{C_{4}, C_{6}, \ldots, C_{2 \ell}\right\}\right)=\Theta\left(n^{\beta_{\ell}(T)}\right) .
$$


The lower bound in Theorem 8 is given as follows. Take an induced subgraph of $T$ consisting of $\beta_{\ell}(T)$ components as described in Definition 3. Replace each path (including the ones of length 0$)$ by $\Omega(n)$ paths of the same length with endpoints joined to the same neighbors as the corresponding paths in $T$ and number of vertices summing to $n$. The resulting graph has $\Omega\left(n^{\beta_{\ell}(T)}\right)$ copies of $T$ and contains no cycle in the set $\left\{C_{4}, C_{6}, \ldots, C_{2 \ell}\right\}$. In fact, we believe that a construction of this form should yield the correct asymptotic value of $\operatorname{ex}_{\mathcal{P}}\left(n, T,\left\{C_{4}, C_{6}, \ldots, C_{2 \ell}\right\}\right)$, but our proof only yields the order of magnitude. We also have the following exact result for maximizing the number of $C_{5}$ copies in a $C_{4}$-free planar graph.

Theorem 9. For all $n \geqslant 4, n \neq 6$, we have

$$
\exp _{\mathcal{P}}\left(n, C_{5},\left\{C_{4}\right\}\right)=n-4 .
$$

Moreover, we determine the order of magnitude of $\operatorname{ex}_{\mathcal{P}}\left(n, C_{k},\left\{C_{4}\right\}\right)$ for every $k$. We obtain the following result.

Theorem 10. For all $k \geqslant 5$, we have

$$
\operatorname{ex}_{\mathcal{P}}\left(n, C_{k},\left\{C_{4}\right\}\right)=\Theta\left(n^{\lfloor k / 3\rfloor}\right) .
$$

We conjecture that in fact a much more general result holds.

Conjecture 11. For sufficiently large $k$, we have

$$
\operatorname{ex}_{\mathcal{P}}\left(n, C_{k},\left\{C_{4}, C_{6}, \ldots, C_{2 \ell}\right\}\right)=\Theta\left(n^{\left\lfloor\frac{k}{\ell+1}\right\rfloor}\right) .
$$

A construction for a lower bound in Conjecture 11 is similar to that of Theorem 8 . Namely, we note that $\beta_{\ell}\left(C_{k}\right)=\left\lfloor\frac{k}{\ell+1}\right\rfloor$, and replace each of the $\beta_{\ell}\left(C_{k}\right)$ paths with $\Omega(n)$ paths of the same length joined to the corresponding pair of vertices. The proof of Theorem 10 can be adapted to resolve Conjecure 11 in the cases when $k$ is congruent to 0,1 or 2 modulo $\ell+1$.

We conclude this section by contrasting our results in the planar case with the known results in the general case. It was shown in [15] and [13] that $\operatorname{ex}\left(n, C_{k}, C_{4}\right)=\Theta\left(n^{k / 2}\right)$. This result is in stark contrast to our results in the planar case in two ways. First, in the planar case the order of magnitude is always an integer power of $n$, and second in the planar case we have $k / 3$ rather than $k / 2$ in the exponent.

\section{General Upper Bounds for Degenerate Graph Classes}

Alon and Shikhelman [2] proved that for any bipartite graph $H$ and tree $T$ we have $\operatorname{ex}(n, H, T)=O\left(n^{\alpha(H)}\right)$, where $\alpha(H)$ is the independence number of $H$. This result was extended to all graphs $H$ in [20]. Since the extremal number, $\operatorname{ex}(n, T)$, of a any tree $T$ is linear in $n$, it follows that any $T$-free graph has a vertex of degree at most $c_{T}$, a constant depending on $T$. We say that a graph $G$ is $c$-degenerate if every subgraph of $G$ contains 
a vertex of degree at most $c$. The proof from [20] can easily be extended to work for the class of $c$-degenerate graphs. We now present a proof of this theorem for completeness.

First we introduce some notation. For given graphs $G$ and $H$, let $\mathcal{N}(H, G)$ denote the number of copies of $H$ in $G$. Let $\mathcal{G}_{c}$ denote the class of $c$-degenerate graphs, and let

$$
f_{c}(n, H):=\max \left\{\mathcal{N}(H, G): G \in \mathcal{G}_{c}, v(G)=n\right\} .
$$

Proposition 12. $f_{c}\left(n, K_{r}\right)=O(n)$, where the hidden constant depends only on $r$ and $c$.

Proof. We proceed by induction on $r$. For $r=1$ the result is clear, so assume $r>1$ and that $f_{c}\left(n, K_{r-1}\right) \leqslant C_{r-1} n$ for a constant $C_{r-1}$. Let $G$ be an $n$-vertex graph in $\mathcal{G}_{c}$, then we have that

$$
r \mathcal{N}\left(K_{r}, G\right)=\sum_{v \in V(G)} \mathcal{N}\left(K_{r-1}, G[N(v)]\right) \leqslant \sum_{v \in V(G)} C_{r-1} d(v)=O(e(G))=O(n) .
$$

We will also make use of the following well-known fact.

Proposition 13. Let $H$ be a graph, and let $u$ be a vertex of $H$. If $H^{\prime}$ is the graph obtained from $H$ by removing $u$ together with its neighborhood, then $\alpha\left(H^{\prime}\right) \leqslant \alpha(H)-1$.

Proof. If $X$ is a maximal independent set in $H^{\prime}$, then since no neighbor of $u$ is in $X$, the set $X \cup\{u\}$ is independent in $H$ and so $\alpha\left(H^{\prime}\right)+1 \leqslant \alpha(H)$.

To prove Theorem 5 we proceed by induction on $\alpha(H)$. The base case when $\alpha(H)=1$ follows from Proposition 12 (or by the results in $[1,35]$ ). Now consider an arbitrary graph $H$ and assume the theorem holds for graphs with independence number less than $\alpha(H)$. The theorem will follow immediately from the following claim.

\section{Claim 14.}

$$
f_{c}(n+1, H)-f_{c}(n, H)=O\left(n^{\alpha(H)-1}\right) .
$$

Here, the constant hidden in the $O$ notation depends only on $H$ and $c$.

Proof of Claim 14. We proceed by induction on $\alpha(H)$, the case when $\alpha(H)=1$ is trivial. To estimate $f_{c}(n+1, H)-f_{c}(n, H)$, we will start with a graph $G \in \mathcal{G}_{c}$ on $n+1$ vertices with the maximum number of copies of $H$. We know that $\delta(G) \leqslant c$. Let $v$ be a vertex of minimum degree in $G$. We will estimate the number of copies of $H$ in $G$ containing $v$.

Let $V(H)=\left\{u_{1}, u_{2}, \ldots, u_{v(H)}\right\}$, and let $H_{i}$ be the graph obtained by removing $u_{i}$ together with its neighbors. By Proposition 13, we know that $\alpha\left(H_{i}\right) \leqslant \alpha(H)-1$. Now for each copy of $H$ using $v$, the vertex $v$ must play the role of some $u_{i}$, and the neighbors of $u_{i}$ must be embedded in the neighborhood of $v$. It follows that the other vertices of $H$, that is the vertices of $H_{i}$, must be embedded in some way in the remaining vertices of $G$. We have to choose $d_{H}\left(u_{i}\right)$ vertices in $N(v)$, so the number of copies of $H$ using $v$ is at most

$$
\sum_{i=1}^{v(H)} d(v)^{d_{H}\left(u_{i}\right)} \mathcal{N}\left(H_{i}, G\right) \leqslant \sum_{i=1}^{v(H)} c^{d_{H}\left(u_{i}\right)} \mathcal{N}\left(H_{i}, G\right)=\sum_{i=1}^{v(H)} O_{H_{i}}\left(n^{\alpha\left(H_{i}\right)}\right)=O\left(n^{\alpha(H)-1}\right) .
$$


Here we have used the notation $O_{H_{i}}$ to emphasize that the constant may depend on the graph $H_{i}$. The second to last equality follows by induction and the last one follows from Proposition 13.

Thus, if $G^{\prime}$ is the graph obtained from $G$ by removing $v$, we have that

$$
f_{c}(n+1, H)=\mathcal{N}(H, G)=\mathcal{N}\left(H, G^{\prime}\right)+O\left(n^{\alpha(H)-1}\right) \leqslant f_{c}(n, H)+O\left(n^{\alpha(H)-1}\right) .
$$

\section{The Number of Trees in Planar Graphs}

In this section we prove Theorem 4. First we provide the lower bound, $\operatorname{ex}_{\mathcal{P}}(n, T, \varnothing)=$ $\Omega\left(n^{\beta(T)}\right)$. Observe that, if a given graph is planar and one blows up a set of independent vertices each of degree at most two, then resulting graph is also planar. Therefore the following construction provides the desired lower bound. Given a tree $T$, fix an independent set $S$ of size $\beta(T)$ which contains vertices of degree at most two (as in Definition 3 ) and blow up this set by $\left\lfloor\frac{n}{2 \beta(T)}\right\rfloor$. The resulting graph is planar with at most $n$ vertices, when $n$ is sufficiently large, and contains $\Omega\left(n^{\beta(T)}\right)$ copies of the tree $T$.

Observe that we have $\operatorname{ex}_{\mathcal{P}}\left(n, P_{k}, \varnothing\right)=O\left(n^{\alpha\left(P_{k}\right)}\right)$ from Theorem 5 , where $P_{k}$ is a $k$ vertex path. Even more we have $\alpha\left(P_{k}\right)=\beta\left(P_{k}\right)$ from Definition 3 . Therefore we have the following simple proposition.

Proposition 15. $\operatorname{ex}_{\mathcal{P}}\left(n, P_{k}, \varnothing\right)=\Theta\left(n^{\beta\left(P_{k}\right)}\right)$.

Hence Theorem 4 holds for paths. To show that Theorem 4 holds for any tree, we are going to use the following lemma.

Lemma 16. For a given planar graph $G$, let $v, u$ and $w$ be fixed vertices in $G$ and let $n_{1}$, $n_{2}$ and $n_{3}$ be nonnegative integers. The number of vertices $x$, such that, there are three internally vertex disjoint paths from $x$ to $v$, from $x$ to $u$ and from $x$ to $w$ of length $n_{1}, n_{2}$ and $n_{3}$, respectively, is bounded by a constant $C:=C\left(n_{1}, n_{2}, n_{3}\right)$.

Proof. Suppose we have a planar embedding of $G$.

The proof will be by induction on $n_{1}+n_{2}$. The result is trivial if either $n_{1}$ or $n_{2}$ is equal to 0 . So suppose that $n_{1}+n_{2} \geqslant 2$, and that the result holds for any pair with smaller sum. Consider a maximal set $\mathcal{P}$, of internally vertex disjoint paths $v, v_{2}^{i}, \ldots, v_{n_{1}}^{i}, a_{i}, u_{2}^{i}, \ldots, u_{n_{2}}^{i}, u$, where each $a_{i}$ is such that there exists a length $n_{3}$ path from $a_{i}$ to $w$ which does not contain any of the vertices $v, v_{2}^{i}, \ldots, v_{n_{1}}^{i}, u_{n_{2}}^{i}, \ldots, u_{2}^{i}, u$. Let us denote the set of $a_{i}$ in these paths by $A$. Observe that the paths from $\mathcal{P}$ divide the plane into $|A|$ regions $R_{1}, R_{2}, \ldots, R_{|A|}$. Since the vertex $w$ is fixed, it is in one of the regions, and there is a path of length $n_{3}$ from $w$ to each vertex of $A$, not using the vertices $v$ and $u$. Thus $|A| \leqslant 2 n_{3}+1$.

Now let $Y$ to be the set of $|A|\left(n_{1}+n_{2}-1\right)+2$ vertices that appear in some path from $\mathcal{P}$, and let $X$ be the set of those vertices $x$ in $G$ which are not in $Y$ such that there exist three internally vertex disjoint paths from $x$ to $v$, from $x$ to $u$ and from $x$ to $w$ of length $n_{1}, n_{2}$ and $n_{3}$, respectively. It is sufficient to bound $|X|+|Y|$ by a constant depending 
on $n_{1}, n_{2}$ and $n_{3}$. If $X=\varnothing$ we immediately have the required bound. Suppose $X$ is nonempty and let $x \in X$, and let $P_{1}=v, v_{2}, \ldots, v_{n_{1}}, x$ and $P_{2}=x, u_{n_{2}}, \ldots, u_{2}, u$ be two of the three internally vertex disjoint paths from $x$. Let $v^{\prime}$ and $u^{\prime}$ be the first vertex (closest to $x$ in $P_{i}$ ) in the intersection of $Y$ with $P_{1}$ and $P_{2}$, respectively. Note that it is possible for $v^{\prime}$ to be $v$ or $u^{\prime}$ to be $u$, but by the definition of $Y$ and $\mathcal{P}$, it is not possible for both to happen simultaneously. Then the vertex $x$ is such that there exist three internally vertex disjoint paths from $x$ to $v^{\prime}$, from $x$ to $u^{\prime}$ and from $x$ to $w$ of length $n_{1}^{\prime}, n_{2}^{\prime}$ and $n_{3}$ respectively, where $1 \leqslant n_{i}^{\prime} \leqslant n_{i}$ for $i=1,2$ and $1 \leqslant n_{3}$, with the additional property that $n_{1}^{\prime}+n_{2}^{\prime}<n_{1}+n_{2}$. Therefore, setting $C^{\prime}=\max _{n_{1}^{\prime}+n_{2}^{\prime}<n_{1}+n_{2}} C\left(n_{1}^{\prime}, n_{2}^{\prime}, n_{3}\right)$, we have that

$$
|X| \leqslant\left(\begin{array}{c}
|A|\left(n_{1}+n_{2}-1\right)+2 \\
2
\end{array}\right) C^{\prime} \leqslant\left(\begin{array}{c}
\left(2 n_{3}+1\right)\left(n_{1}+n_{2}-1\right)+2 \\
2
\end{array}\right) C^{\prime}
$$

Thus, $|X|+|Y|$ is bounded and so the lemma holds.

Note that Lemma 16 implies in particular that if $G$ is a planar graph and $T$ is a tree with $s \geqslant 3$ leaves $x_{1}, x_{2}, \ldots, x_{s}$, then for any vertex $x \in V(T)$ of degree at least 3 and $v_{1}, v_{2}, \ldots, v_{s} \in V(G)$, the number of vertices $v \in V(G)$, such that, there exists a copy of $T$ where $x$ is embedded at $v$ and $x_{i}$ is embedded at $v_{i}$, for $i=1, \ldots, s$, is bounded by a constant that does not depend on $G$. This follows since we are able to find three different leaves such that the paths from $x$ to each of these leaves are internally vertex disjoint.

At this point we are ready to prove Theorem 4.

Proof of Theorem 4. We may assume $T$ is not a path otherwise we are done, from Proposition 15 .

Let $G$ be an $n$-vertex planar graph. Let $A$ be the set of vertices of degree at least 3 in $T$, and let $T_{1}, T_{2}, \ldots, T_{k}$ be the connected components of the graph induced by $V(T) \backslash A$ (the set $A$ is non-empty since $T$ is not a path). Observe that since $A$ has every vertex of degree at least 3 , then every vertex of $T_{i}$ has degree at most 2 in both $T_{i}$ and $T$, so we have

$$
\beta(T)=\beta\left(\bigcup_{i=1}^{k} T_{i}\right)=\sum_{i=1}^{k} \beta\left(T_{i}\right) .
$$

Moreover the trees $T_{i}$ are paths and so $\mathcal{N}\left(T_{i}, G\right)=O\left(n^{\beta\left(T_{i}\right)}\right)$, from Proposition 15. Then for any embedding of the trees $T_{i}$ by Lemma 16 , there is a constant number of ways to complete the embedding of the set $A$ to a copy of $T$. Therefore the number of copies of $T$ is bounded by $O\left(n^{\sum_{i=1}^{k} \beta\left(T_{i}\right)}\right)=O\left(n^{\beta(T)}\right)$.

\section{The number of $C_{5}$ 's in $C_{4}$-free Planar Graphs}

In this section we are going to prove Theorem 9 , namely that for all $n, n \geqslant 4, n \neq 6$, we have

$$
\operatorname{ex}_{\mathcal{P}}\left(n, C_{5},\left\{C_{4}\right\}\right)=n-4
$$


Proof. We begin by providing the lower bound. Let $n=5+3 t+2 s$ for some nonnegative integers $s$ and $t$. (Note that when $n=6$, it is easy to verify that there can be at most one pentagon, thus $\operatorname{ex}_{\mathcal{P}}\left(6, C_{5},\left\{C_{4}\right\}\right)=1$.) The construction is as follows: Take a pentagon $x_{1}, x_{2}, x_{3}, x_{4}, x_{5}$, as well as $t$ internally vertex disjoint paths $x_{1}, y_{3}^{i}, y_{4}^{i}, y_{5}^{i}, x_{2}$ (where $1 \leqslant i \leqslant t$ and $y_{j}^{i} \notin\left\{x_{3}, x_{4}, x_{5}\right\}, j \in[3]$ ) between $x_{1}$ and $x_{2}$ and add the edges so that $x_{4}, y_{4}^{1}, \ldots, y_{4}^{t}$ forms a path. Next take a path $z_{1}, z_{2}, \ldots, z_{2 s}$ on new set of vertices. Add the edge from $z_{1}$ to $x_{1}$ and the edges from $z_{i}$ to $x_{5}$, for odd $i \equiv 0,1(\bmod 4)$, and $z_{i}$ to $x_{3}$, for $i \equiv 2,3(\bmod 4)$. See Figure 1 for an example of an extremal graph.

Observe that the 5-cycles in the construction are either $x_{1}, x_{2}, x_{3}, x_{4}, x_{5}$ or the 5 -cycles containing $y_{4}^{i}, 1 \leqslant i \leqslant t$ or the 5 -cycles containing an edge $\left\{z_{i}, z_{i+1}\right\}, 1 \leqslant i \leqslant s$ and $i \equiv 1,3(\bmod 4)$.

For $1 \leqslant i \leqslant t$, we have three distinct 5 -cycles containing $y_{3}^{i}$ in the construction. These are $x_{2}, y_{3}^{i}, y_{4}^{i}, y_{4}^{i-1}, y_{3}^{i-1}$ or $x_{1}, y_{5}^{i}, y_{4}^{i}, y_{4}^{i-1}, y_{5}^{i-1}$ or $x_{2}, y_{3}^{i}, y_{4}^{i}, y_{5}^{i}, x_{1}, x_{2}$, where $y_{3}^{i-1}, y_{4}^{i-1}$ and $y_{5}^{i-1}$ are respectively $x_{3}, x_{4}$ and $x_{5}$ when $i=1$. Thus we have $3 t 5$-cycles in the construction containing $y_{4}^{i}, 1 \leqslant i \leqslant t$.

On the other hand for $1 \leqslant i \leqslant s$ and $i \equiv 1,3(\bmod 4)$, each edge $\left\{z_{i}, z_{i+1}\right\}$ is contained in two distinct 5 -cycles of the construction. Indeed, for an edge $\left\{z_{i}, z_{i+1}\right\}(i \equiv 1(\bmod 4))$, notice that $z_{i+1}$ and $z_{i-2}$ are adjacent with $x_{3}$ and $z_{i}$ is adjacent with $x_{5}$. Hence we have the 5 -cycles $x_{3}, z_{i+1}, z_{i}, x_{5}, x_{4}$ and $x_{3}, z_{i+1}, z_{i}, z_{i-1}, z_{i-2}$ which contain the edge $\left\{z_{i}, z_{i+1}\right\}$. In the case that $i=1$, we take $x_{1}$ and $x_{2}$ in place of $z_{i-1}$ and $z_{i-2}$ respectively. For an edge $\left\{z_{i}, z_{i+1}\right\}(i \equiv 3(\bmod 4))$, observe that $z_{i+1}$ and $z_{i-2}$ are adjacent with $x_{5}$ and $z_{i}$ is adjacent with $x_{3}$. In this scenario, we have two distinct 5 -cycles containing the edge $\left\{z_{i}, z_{i+1}\right\}$, namely $x_{5}, z_{i+1}, z_{i}, z_{i-1}, z_{i-2}$ and $x_{5}, z_{i+1}, z_{i}, x_{3}, x_{4}$. Thus, we have $2 s 5$-cycles in the construction containing $\left\{z_{i}, z_{i+1}\right\}$, where $1 \leqslant i \leqslant s$. Therefore, the number of 5-cycles in the construction is $3 t+2 s+1=n-4$.

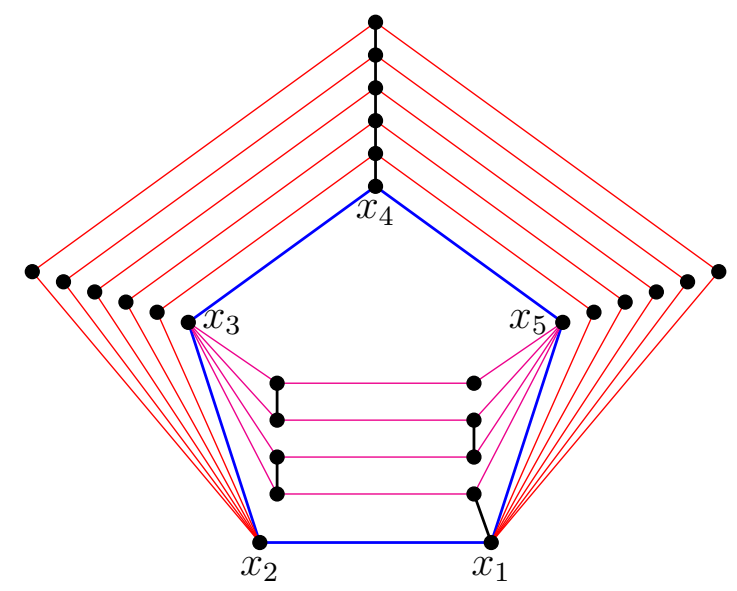

Figure 1: Example of an extremal graph for Theorem 9.

Now we are going to prove by induction that $\operatorname{ex}_{\mathcal{P}}\left(n, C_{5},\left\{C_{4}\right\}\right) \leqslant n-4$. The proof proceeds by induction on $n$ with the base cases $\operatorname{ex}_{\mathcal{P}}\left(4, C_{5},\left\{C_{4}\right\}\right)=0, \operatorname{ex}_{\mathcal{P}}\left(5, C_{5},\left\{C_{4}\right\}\right)=1$ and $\operatorname{ex}_{\mathcal{P}}\left(6, C_{5},\left\{C_{4}\right\}\right)=1$. Let $G$ be an $n$ vertex, $C_{4}$-free planar graph with $n \geqslant 7$. Without 
loss of generality we may assume that $G$ is connected. Consider an embedding of $G$ on the plane. To prove an upper bound for Theorem 9, we take a planar embedding of $G$ and consider two cases.

Case 1: All pentagons in $G$ are face. We can assume that $G$ is a connected plane graph. Otherwise, we can still join the components of $G$ with edges so that there is no new cycle created.

Remove an edge from each triangular face. Observe that two triangular faces do not share an edge since $G$ is $C_{4}$-free. Let us denote the resulting graph by $G^{\prime}$. Since there is no pentagon in $G^{\prime}$ which is not a face, the total number of faces of $G^{\prime}$ is at least the number of pentagonal faces in $G$.

Let $f_{i}$ denote the number of faces of size $i$ in $G^{\prime}$, and let $f$ denote the number of faces in $G$. Since $G^{\prime}$ is connected and $n \geqslant 7, f_{1}=f_{2}=0$. Moreover, since $G^{\prime}$ contains no triangular face and $G^{\prime}$ contains no $C_{4}$ we have $f_{3}=f_{4}=0$. Thus,

$$
2 e\left(G^{\prime}\right)=\sum_{i \geqslant 5} i f_{i}=5 f_{5}+\sum_{i \geqslant 6} i f_{i} \geqslant 5 f_{i}+5\left(f-f_{i}\right)=5 f .
$$

This implies $5 f \leqslant 2 e\left(G^{\prime}\right)$. Using the Euler's formula, $f+n=e\left(G^{\prime}\right)+2$, we get $f \leqslant \frac{2}{3} n-\frac{4}{3}$. Thus we have that the number of $C_{5}$ copies in $G$ is at most $n-4$, since $n \geqslant 7$.

Case 2: There is a pentagon in $G$ which is not a face. Let $P$ be a non-facial pentagon in $G$. The pentagon $P$ cuts the plane into two regions. Let us denote the subgraph of $G$ in the inner and outer regions of $P$ by $G_{1}$ and $G_{2}$, respectively, where both graphs include the vertices of the pentagon. Assume that $G_{1}$ and $G_{2}$ have $n_{1}$ and $n_{2}$ vertices respectively. Thus we have $n=n_{1}+n_{2}-5$. Since $n_{1}$ and $n_{2}$ are both non-zero and less than $n$ and both $G_{1}$ and $G_{2}$ are $C_{4}$-free, then by induction hypothesis, the number of pentagons in $G_{1}$ and $G_{2}$ is at most $n_{1}-4$ and $n_{2}-4$, respectively.

It can be checked that there is no pentagon crossing $P$ in $G$. Indeed, let $P=$ $x_{1}, x_{2}, x_{3}, x_{4}, x_{5}$. Notice that since $G$ is $C_{4}$-free, no two non-consecutive vertices of $P$ are adjacent. Suppose that there is a pentagon, say $P^{\prime}$, crossing $P$. $P^{\prime}$ must contain a cherry $x_{i}, y, x_{j}$ where $y$ is a vertex either in the interior or in the exterior region of $P$. Without loss of generality suppose $y$ is in the interior region of $P$. Notice that since $G$ is $C_{4}$-free, $x_{i}$ and $x_{j}$ can not be non-consecutive vertices of the pentagon $P$. Thus, we assume that $x_{i}$ and $x_{j}$ are adjacent vertices of $P$. Without loss of generality assume that $i=1$ and $j=2$. Since any two non-consecutive vertices of $P$ are not adjacent, there are two vertices say, $y_{1}$ and $y_{2}$ in the exterior region of $P$ such that $P^{\prime}=x_{1}, y, x_{2}, y_{1}, y_{2}$. However, in this case we got a $C_{4}$, namely $x_{1}, x_{2}, y_{1}, y_{2}$, which is a contradiction to the fact that $G$ is $C_{4}$-free.

From the above observation it follows that every pentagon of $G$ is a pentagon of $G_{1}$ or $G_{2}$. Since the pentagon $P$ is in both graphs, we get that the number of pentagons in $G$ is at most $n_{1}-4+n_{2}-4-1=n+5-9=n-4$, completing the proof. 


\section{The Order of Magnitude of the Maximum Number of Cycles of Length $k$ in a $C_{4}$-free Planar Graph}

We have seen in Corollary 6 that $\operatorname{ex}_{\mathcal{P}}\left(n, C_{k}, \varnothing\right)=\Theta\left(n^{\lfloor k / 2\rfloor}\right)$ follows immediately from Theorem 5. We now consider the case when $C_{4}$ is forbidden and prove Theorem 10 which states that $\operatorname{ex}_{\mathcal{P}}\left(n, C_{k},\left\{C_{4}\right\}\right)=\Theta\left(n^{\lfloor k / 3\rfloor}\right)$.

Proof. For the construction we take a cycle $C_{k}$ and find an induced matching of size $\lfloor k / 3\rfloor$. Next we replace each edge in this matching with $\frac{n-k}{2\lfloor k / 3\rfloor}$ edges each adjacent to the same pair of vertices as the original edge. This graph clearly has $\left.\Theta\left(n^{\left\lfloor\frac{k}{3}\right.}\right\rfloor\right)$ copies of $C_{k}$ and at most $n$ vertices, when $n$ is sufficiently large.

We will now prove the upper bound. It is well-known that every planar graph contains a vertex of degree at most 5 . It was proved in [25] that a $C_{4}$-free planar graph with minimum degree at least 2 contains an edge $\{x, y\}$ such that $d(x)+d(y)$ is at most 8. This result was improved to 7 in [5], which is best possible. Note that, in proving Theorem 10, we may delete all edges containing a vertex of degree at most one, since such vertices do not contribute to any $k$-cycles.

We will distinguish cases based on the value of $k$ modulo 3 . When $k$ is equal to 0 or 1 modulo 3, the result can be proved using the fact that a planar graph contains a vertex of degree at most 5 . We present here the proof in the case when $k \equiv 2(\bmod 3)$, the other cases are similar but require only the fact that there is a vertex of bounded degree.

Suppose $k=3 m+2$ for some integer $m \geqslant 1$ and that $G$ is an $n$-vertex, $C_{4}$-free planar graph with no vertex of degree at most 1. Let us label the vertices of $C_{k}$ by $v_{1}, v_{2}, \ldots, v_{3 m+2}$, consecutively. Applying the aforementioned result of [5] we find an edge $\{u, v\}$ such that $d(u)+d(v) \leqslant 7$. We will show that at most $n^{m-1}$ cycles $C_{k}$ can use the edge $\{u, v\}$. Then, by iteratively deleting such an edge edge and the resulting vertices of degree 1, we find all copies of $C_{k}$ after at most $O(n)$ steps (since there are at most linearly many edges in a planar graph). Thus in total we will have shown that there are at most $n^{m}$ copies of $C_{k}$ in $G$, as required.

Let us consider a copy of $C_{k}$ so that $u$ and $v$ correspond to $v_{1}$ and $v_{2}$ in the $C_{k}$. There is at most 7 ways to embed $v_{3 m+2}$ and $v_{3}$ as neighbors of $v_{1}$ and $v_{2}$, respectively. Thus we now consider $v_{3 m+2}, v_{1}, v_{2}$ and $v_{3}$ as being embedded. Next choose edges of $G$ at which to embed the edges of the cycle $\left\{v_{5}, v_{6}\right\},\left\{v_{8}, v_{9}\right\},\left\{v_{11}, v_{12}\right\}$ and so on. We now consider embedding the remaining vertices. Since our graph is $C_{4}$-free, there is at most one way to embed the vertices $v_{4}, v_{7}, v_{10}$ and so on. It follows that we have at most on the order of $n^{m-1}$ copies of the cycle $C_{k}$ in the graph which use the edge $\{u, v\}$, and we are done.

\section{The Number of Trees in Planar Graph with no even cycle of length at most $2 \ell$}

In this section we prove Theorem 8 , namely that

$$
\exp _{\mathcal{P}}\left(n, T,\left\{C_{4}, C_{6}, \ldots, C_{2 \ell}\right\}\right)=\Theta\left(n^{\beta_{\ell}(T)}\right) .
$$


Proof of Theorem 8. First we will show that the result is true for paths. We will make use of the following theorem due to Lam and Verstraete.

Theorem 17 ([27]). Let $G$ be a graph containing no even cycles of length at most $2 \ell$. There exists a constant $D_{\ell}$ such that for any vertices $v, u$ of $G$ and $k \leqslant \ell$ a positive integer, the number of paths from $v$ to $u$ of length $k$ in $G$ is at most $D_{\ell}$.

It is simple to check that $\beta_{\ell}\left(P_{k}\right)=1+\left\lfloor\frac{k+\ell-2}{\ell+1}\right\rfloor$. Now we will prove that

$$
\operatorname{ex}_{\mathcal{P}}\left(n, P_{k},\left\{C_{4}, C_{6}, \ldots, C_{2 \ell}\right\}\right)=O\left(n^{1+\left\lfloor\frac{k+\ell-2}{\ell+1}\right\rfloor}\right) .
$$

Let $G$ be an $n$-vertex planar graph containing no even cycle of length at most $2 \ell$. We will bound the number of $k$-vertex paths in $G$ by fixing a particular $k$-vertex path, $v_{1}, v_{2}, \ldots, v_{k}$, and bounding the number of ways it can be embedded in $G$. Suppose $k \geqslant 3$ (otherwise we are already done), and fix the images of the edges $\left\{v_{(\ell+1) i+1}, v_{(\ell+1) i+2}\right\}$ for $i=0,1, \ldots,\left\lfloor\frac{k+\ell-2}{\ell+1}\right\rfloor-1$ and the edge $\left\{v_{k-1}, v_{k}\right\}$ in the embedding. With these edges embedded, by Theorem 17 there is a constant number of ways to embed the portion the paths between each pair of consecutive edges which we enumerated. We notice that we have a linear number of choices for each of the edges which we specified, and in total the number of choices for these edges is of order $n^{1+\left\lfloor\frac{k+\ell-2}{\ell+1}\right\rfloor}$. Therefore $\mathcal{N}\left(G, P_{k}\right)=O\left(n^{1+\left\lfloor\frac{k+\ell-2}{\ell+1}\right\rfloor}\right)$.

Now let $T$ be any tree. Let us partition the vertex set $V(T)$ into five sets $A_{1}, A_{2}, A_{2}^{\prime}$, $A_{2}^{\prime \prime}$ and $A_{\geqslant 3}$. First we partition the set of vertices of degree not equal to 2 as follows.

$$
A_{1}=\{v \in V(T) \mid d(v)=1\} \text { and } A_{\geqslant 3}=\{v \in V(T) \mid d(v) \geqslant 3\} .
$$

In particular, $A_{1}$ is the set of leaves of the tree $T$. Now we will partition the set of vertices of degree equal to 2 into three sets. Let

$A_{2}=\left\{v \in V(T) \mid d(v)=2\right.$ and there is no vertex of $A_{\geqslant} 3$ at distance less than $\ell$ from $\left.v\right\}$.

Now consider every path $P$ in $T$ such that:

(i) Both end-vertices of $P$ have degree at least 3 in $T$.

(ii) The length of $P$ is at least $\ell+1$, but at most $2 \ell-1$.

(iii) Every internal vertex of $P$ has degree 2 in $T$.

For each such path let $f(P)$ be the middle vertex of $P$; if $P$ has odd length, take either of the two middle vertices.

Let $A_{2}^{\prime}$ be the set of consisting of the vertices $f(P)$ for the paths $P$ defined above. Finally, define

$$
A_{2}^{\prime \prime}=\left\{v \in V(T) \mid d(v)=2, v \notin A_{2} \cup A_{2}^{\prime}\right\} .
$$

Let $F$ be the subgraph of $T$ induced by the vertex set $V_{1}=A_{1} \cup A_{2} \cup A_{2}^{\prime}$. Note that $F$ is a path forest and suppose $F=P_{i_{1}} \cup P_{i_{2}} \cup \cdots \cup P_{i_{t}}$ for paths $P_{i_{j}}, 1 \leqslant j \leqslant t$. 
Now we will show that $\beta_{\ell}(F)=\beta_{\ell}(T)$. Take a set $S$ of vertices and paths which is a witness for the value of $\beta_{\ell}(T)$ in $T$. Suppose $S$ contains a path $P=x_{1}, x_{2}, \ldots, x_{\ell}$ using at least one vertex from $A_{2}^{\prime \prime}$. Note that it is not possible for this path to be fully contained in $A_{2}^{\prime \prime}$. Indeed, if it was contained in $A_{2}^{\prime \prime}$ we would be able to find an $i$ such that $x_{i}$ is at distance $\ell-1$ from a vertex $v \in A_{\geqslant 3}$ and $x_{i+1}$ is at distance $\ell-1$ from a vertex $u \in A_{\geqslant 3}$ and so $P$ would be contained in a path of length $2 \ell+1$ from $v$ to $u$. It follows that the middle vertex of this path must be a vertex of $P$. So we may replace the choice of $P$ in $S$ with a vertex (leaf) $f(P)$ in $F$. We obtain that $\beta_{\ell}(F) \geqslant \beta_{\ell}(T)$.

Next we show $\beta_{\ell}(F) \leqslant \beta_{\ell}(T)$. Take a set $S$ of vertices and paths which is a witness for $\beta_{\ell}(F)$ in $F$. If $P$ is a length $\ell$ path in $S$, by definition, no end-vertex is a leaf in $F$, so $P$ is still a length $\ell$ path in $T$ such that every vertex has degree 2 . If $v$ is a leaf in $F$, but is no longer a leaf in $T$, then $v$ must have degree 2 in $T$, and there are two possibilities. Suppose $v \in A_{2}^{\prime \prime}$, then there exists a path $P$ of length at least $\ell+1$ with internal vertices of degree 2 containing $v$. In this case we may replace the choice of $v$ in $S$ by a subpath of $P$ of length $\ell-1$ without using the end-vertices of $P$. Suppose $v$ is in $A_{2}$, since one neighbor of $v$ is not in $F$, it must be in $A_{\geqslant 3} \cup A_{2}^{\prime \prime}$, but by definition of $A_{2}$, then $v$ must have a neighbor in $A_{2}$ and so $v$ is at distance $\ell$ of $A_{\geqslant 3}$. Let $u$ be the closest neighbor of $v$ in $u$, and let $P$ be the length $\ell$ path from $v$ to $u$, then we may replace the choice of $v$ in $S$ by $P^{\prime}$ the path obtained from $P$ by deleting $u$. It follows that $\beta_{2}(F) \leqslant \beta_{2}(T)$.

Now let $G$ be an $n$-vertex planar graph containing no even cycle of length at most $2 \ell$ and fix a copy of $F$ in $G$. By Lemma 16 since every leaf of $T$ is already fixed, we have a bounded number of choices to embed the vertices of $A_{\geqslant 3}$ in $G$ such that together with the copy of $F$ the embedding can be completed to a copy of $T$. For any given embedding of $F$ and $A_{\geqslant 3}$, let $x \in A_{2}^{\prime \prime}$. By the definition of $A_{2}^{\prime \prime}$, there is a vertex $a \in A_{\geqslant 3}$ with distance less than $\ell$ to $x$. If there are two choices for $a$, pick one which is closest to $x$, on the branch from $x$ that does not contain $a$, and let $b$ be the closest vertex of $V \backslash A_{2}^{\prime \prime}$. We show that the distance between $a$ and $b$ is at most $\ell$. Suppose by contradiction $b$ is at distance more than $\ell$ from $a$, then pick $c$ to be the vertex between $x$ and $b$ at distance exactly $\ell$ from $a$. It follows that $c \in A_{2}^{\prime \prime}$, so there is a vertex $d \in A_{\geqslant 3}$ in the same branch from $x$ as $b$ and $c$, at distance at most $\ell$ from $c$. Hence the path from $a$ to $d$ has length at most $2 \ell-1$ and its middle vertex $y$ is in $F$ and it is closer to $x$ than $y$, but this contradicts the choice of $b$ since $y$ is also in the branch from $x$ not containing $a$. By Theorem 17, there is a constant number of choices to embed $x$ in $G$ such that the embedding can be completed to $T$, since for each such embedding we have a path of length at most $\ell$ from the vertices corresponding to $a$ and $b$ in $G$.

\section{Acknowledgements.}

We thank Kevin Hendrey for providing a reference to the fourth author for the fact that a $C_{4}$-free planar graph with no leaf contains an edge whose degree sum is bounded by a constant. We also thank Tony Huynh for providing us with the reference [10]. We also thank the anonymous referee for their valuable remarks. 


\section{References}

[1] N. Alon, Y. Caro. On the Number of Subgraphs of Prescribed Type of Planar Graphs With a Given Number of Vertices. Annals of Discrete Mathematics 20 (1984): 25-36.

[2] N. Alon, C. Shikhelman. Many $T$ copies in $H$-free graphs. Journal of Combinatorial Theory, Series B 121 (2016): 146-172.

[3] N. Alon, C. Shikhelman. Additive Approximation of Generalized Turán Questions. arXiv: 1811.08750 (2018).

[4] B. Bollobás, E. Győri. Pentagons vs. triangles. Discrete Mathematics 308.19 (2008): 4332-4336.

[5] O. Borodin, A. Kostochka, N. Sheikh, G. Yu. M-degrees of quadrangle-free planar graphs. Journal of Graph Theory 60(1) (2009): 80-85.

[6] G. Chen, Y. Lan and Z. Song. Planar anti-Ramsey numbers of matchings. Discrete Mathematics 342.7 (2019): 2106-2111.

[7] C. Cox and R. R. Martin. Counting paths, cycles and blow-ups in planar graphs. arXiv:2101.05911v1 (2021).

[8] C. Cox and R. R. Martin. The maximum number of 10-and 12-cycles in a planar graph. arXiv:2106.02966 (2021).

[9] C. Dowden. Extremal $C_{4}$-Free $/ C_{5}$-Free Planar Graphs. Journal of Graph Theory 83.3 (2016): 213-230.

[10] D. Eppstein. Connectivity, graph minors, and subgraph multiplicity. Journal of Graph Theory 17.3 (1993): 409-416.

[11] P. Erdös. On the number of complete subgraphs contained in certain graphs. Magyar Tud. Akad. Mat. Kut. Int. Közl. (1962): 459-474.

[12] P. Erdős. On some problems in graph theory, combinatorial analysis and combinatorial number theory. Graph Theory and Combinatorics (Cambridge, 1983), Academic Press, London (1984): 1-17.

[13] D. Gerbner, E. Győri, A. Methuku, M. Vizer. Generalized Turán problems for even cycles. Journal of Combinatorial Theory, Series B 145 (2020): 169-213.

[14] D. Gerbner, C. Palmer. Counting copies of a fixed subgraph in F-free graphs. European Journal of Combinatorics 82 (2019): 103001.

[15] Gishboliner, Lior, and Asaf Shapira. A generalized Turán problem and its applications. Proceedings of the 50th Annual ACM SIGACT Symposium on Theory of Computing (2018).

[16] D. Ghosh, E. Győri, R.R. Martin, A. Paulos, N. Salia, C. Xiao, O. Zamora. The maximum number of paths of length four in a planar graph. Discrete Mathematics 344 (2021): 112317.

[17] A. Grzesik. On the maximum number of five-cycles in a triangle-free graph. Journal of Combinatorial Theory, Series B 102(5), (2012): 1061-1066.

[18] E. Győri, A. Paulos, N. Salia, C. Tompkins, O. Zamora. The Maximum Number of Pentagons in a Planar Graph. arXiv:1909.13532 (2019). 
[19] E. Győri, A. Paulos, N. Salia, C. Tompkins, O. Zamora. The Maximum Number of Paths of length three in a Planar Graph. arXiv:1909.13539 (2019).

[20] E. Győri, N. Salia, C. Tompkins, O. Zamora. The maximum number of $P_{\ell}$ copies in a $P_{k}$-free graph. Discrete Mathematics \& Theoretical Computer Science 21(1) arXiv: 1803.03240 (2019).

[21] S. Hakimi, E.F. Schmeichel. On the Number of Cycles of Length $k$ in a Maximal Planar Graph. J. Graph Theory 3 (1979): 69-86.

[22] H. Hatami, J. Hladký, D. Král, S. Norine, A. Razborov. On the number of pentagons in triangle-free graphs. Journal of Combinatorial Theory, Series A 120(3) (2013): $722-732$.

[23] T. Huynh, G. Joret, D.R. Wood. Subgraph densities in a surface. arXiv:2003.13777 (2020).

[24] T. Huynh, D.R. Wood. Tree densities in sparse graph classes. arXiv:2009.12989 (2020).

[25] W. He, X. Hou, K. Lih, J. Shao, W. Wang, X. Zhu. Edge-partitions of planar graphs and their game coloring numbers, J. Graph Theory 41 (2002): 307-317.

[26] K. Kuratowski. Sur le probléme des courbes gauches en topologie. Fund. Math. (in French) 15 (1930): 271-283.

[27] T. Lam, J. Verstraete. A note on graphs without short even cycles. The Electronic Journal of Combinatorics 12(1) (2005), \#N5.

[28] Y. Lan and Y. Shi. Planar Turán Numbers of Short Paths. Graphs and Combinatorics (2019): 1-15.

[29] Y. Lan, Y. Shi, Z. Song. Extremal $H$-free planar graphs. arXiv: 1808.01487 (2018).

[30] Y. Lan, Y. Shi, Z. Song. Extremal Theta-free planar graphs. Discrete Mathematics $342.12(2019): 111610$.

[31] Y. Lan, Y. Shi and Z. Song. Planar anti-Ramsey numbers of paths and cycles. Discrete Mathematics 342.11 (2019): 3216-3224.

[32] S. Letzter. Many $H$-copies in graphs with a forbidden tree. SIAM Journal on Discrete Mathematics 33.4 (2019): 2360-2368.

[33] R. Luo. The maximum number of cliques in graphs without long cycles. Journal of Combinatorial Theory, Series B 128 (2018): 219-226.

[34] D. Matolcsi, Z.L. Nagy. Generalized Outerplanar Turán numbers and maximum number of k-vertex subtrees. arXiv:2102.11746 (2021).

[35] D. Wood. On the maximum number of cliques in a graph. Graphs and Combinatorics 23.3 (2007): 337-352.

[36] N. Wormald. On the frequency of 3-connected subgraphs of planar graphs. Bulletin of the Australian Mathematical Society 34.2 (1986): 309-317.

[37] K. Zarankiewicz. Problem p 101. Colloq. Math. 2(1951): 116-131.

[38] A. Zykov. On some properties of linear complexes. Mat. Sbornik N. S. 24(66) (1949): $163-188$. 